\title{
Will the revolution in genetics improve healthcare?
}

\author{
We need randomised trials to tell us
}

\author{
Liam Smeeth professor of clinical epidemiology ${ }^{1}$, Tjeerd van Staa director of research ${ }^{2}$ \\ 'London School of Hygiene and Tropical Medicine, London WC1E 7HT, UK; ${ }^{2}$ Utrecht Institute for Pharmaceutical Sciences, Utrecht University, \\ Utrecht, Netherlands
}

The new millennium has brought with it an explosion in genetic knowledge. Future generations are likely to look back at this time as the beginning of a new era in human genetics and health. The major challenge facing researchers is how to translate this new knowledge into improved health and healthcare, and progress has been slower than predicted. ${ }^{12}$ Simply knowing that a genetic variant is associated with an increased risk of a disease or that it influences a response to drug treatment is only half the picture. The new research frontiers are how this knowledge should be used and assessing the clinical and cost effectiveness of such use. A linked research study by Hollands and colleagues (doi:10.1136/bmj.e4708) is a pioneering attempt to use a genetic discovery to improve healthcare. ${ }^{3}$

Increased knowledge of the underlying genetic causes of disease has already made major contributions. For example, the finding that genetic variants of components of the complement pathway were associated with age related macular degeneration stimulated a whole new area of research and suggested possible drug targets for what had been a largely untreatable disease. ${ }^{4}$ Improvement in knowledge is sufficient justification for this kind of genetic research. Immediate health gain is not the aim: the hope is for translation into improved health at some time in the future.

However, for two other major areas of genetic research-prediction of disease risk and pharmacogenetics-improvements in health may be more immediate. In the past decade genetic variants that influence a range of common multifactorial disorders or that affect response to drug treatment have been identified reliably. However, current knowledge explains only a small proportion of the heritability of most diseases. ${ }^{5}$ The genetic basis for many adverse drug reactions is unknown, with even less known about genetic factors associated with poor response to treatment. ${ }^{6}$ Continued research aimed at understanding how genes influence human health is clearly needed.

Hollands and colleagues' study was based on the association between the NOD2 gene (which encodes nucleotide binding oligomerisation domain containing protein 2) and Crohn's disease. First degree relatives of people with Crohn's disease have a higher risk of the disease compared with the general population. This increased risk is enhanced by the presence of NOD2 mutations, with greater risk being conferred by two mutations than by one. Smoking is an established causal risk factor for Crohn's disease, and quitting decreases the risk of the disease. The main hypothesis tested in the study was that informing people of their genetic risk of Crohn's disease on the basis of their NOD2 genotype would motivate them to stop smoking. Crucially, the authors not only implemented the use of genetic knowledge but they evaluated the effectiveness of using the knowledge in a randomised trial. The comparison group was offered non-DNA based risk information. All participants were smokers who had first degree relatives with Crohn's disease and thus all had a high risk of the disease.

Although the study was negative- - smoking quit rates did not differ between the two arms- the authors are to be applauded for undertaking a rigorous randomised trial in an area where strong evidence is lacking. However, their conclusion that genetic knowledge of disease risk does not motivate behaviour change may be too sweeping given aspects of the study that could have contributed to the negative result. People's genetic risk was communicated by post in the form of a booklet. Although a smoking cessation counsellor made a follow-up call, face to face communication of risk may have been more effective. Only 50 of 209 people (24\%) in the intervention group had one or more NOD2 mutations, so any impact would have been restricted to these 50 people, which compromised the statistical power of the study to detect a beneficial effect. But, perhaps most importantly, the increased risk of Crohn's disease conferred by $N O D 2$ variants (ranging from double to sevenfold) is dwarfed by the massively increased risk (20-fold) in all participants through them having a first degree relative with the disease. The incremental effect of knowing NOD2 status might thus be expected to be small.

In spite of these limitations, Hollands and colleagues' study is a welcome and important addition to the literature because it used the most reliable and robust tool to assess effectiveness: the randomised trial. Knowledge of genetics will continue to increase rapidly, particularly as the use of whole genome sequencing becomes more widespread. Improved prediction of risk and better targeting of drugs are realistic expectations. 
However, new technologies and models of care that are based on genetic information need to be rigorously evaluated before they are implemented. The optimal test will be a randomised trial comparing care that does and does not use genetic knowledge. Typically trials will test interventions tailored to individuals according to a composite of their personal genetic information along with other clinical factors. Such trials are needed to tell us which aspects of healthcare can be improved by the revolution in genetics.

Competing interests: Both authors have completed the ICMJE uniform disclosure form at www.icmje.org/coi_disclosure.pdf (available on request from the corresponding author) and declare: LS was supported by a senior clinical fellowship from the Wellcome Trust; no financial relationships with any organisations that might have an interest in the submitted work in the previous three years; no other relationships or activities that could appear to have influenced the submitted work.
Provenance and peer review: Commissioned; not externally peer reviewed.

1 Hingorani AD, Shah T, Kumari M, Sofat R, Smeeth L. Translating genomics into improved healthcare. BMJ 2010;341:c5945.

2 Lander ES. Initial impact of the sequencing of the human genome. Nature 2011;470:187-197.

3 Hollands GJ, Whitwell SCL, Parker RA, Prescott NJ, Forbes A, Sanderson J, et al. Effect of communicating DNA based risk assessments for Crohn's disease on smoking cessation: randomised controlled trial. BMJ 2012;345:e4708.

4 Hageman GS, Anderson DH, Johnson LV, Hancox LS, Taiber AJ, Hardisty LI, et al. A common haplotype in the complement regulatory gene factor $\mathrm{H}$ (HF1/CFH) predisposes individuals to age-related macular degeneration. Proc Natl Acad Sci USA 2005; 102:7227-32.

5 Goldstein DB. Common genetic variation and human traits. N Engl J Med 2009;360:1696-8.

6 Holmes MV Shah T, Vickery C, Smeeth L, Hingorani AD, Casas JP. Fulfilling the promise of personalized medicine? Systematic review and field synopsis of pharmacogenetic studies. PloS One 2009;4:e7960.

Cite this as: BMJ 2012;345:e4651

๑ BMJ Publishing Group Ltd 2012 